\title{
Gut microbes ward off obesity
}

Microbes in the gut can greatly affect host physiology and disease. They can locally influence the development of immune cells and distally affect systems by metabolizing dietary components and releasing factors into the circulation. Several studies have suggested that diet profoundly influences the composition of the bacterial community in the gut, termed the microbiota. Altered composition of the microbiota is found in obese individuals, and germ-free mice that lack gut microbes are protected from diet-induced obesity and insulin resistance.

To assess how these microbes interact with diets and metabolic disease, Robert Caesar, Fredrik Bäckhed and colleagues at the University of Gothenburg (Cell Metab. doi:10.1016/j.cmet.2015.07.026; published online 27 August 2015) fed mice diets that were rich in either lard (saturated lipids) or fish oil (polyunsaturated lipids). As was expected, mice that were fed lard gained much weight and developed insulin resistance, whereas mice that were fed fish oil did not develop metabolic disease.

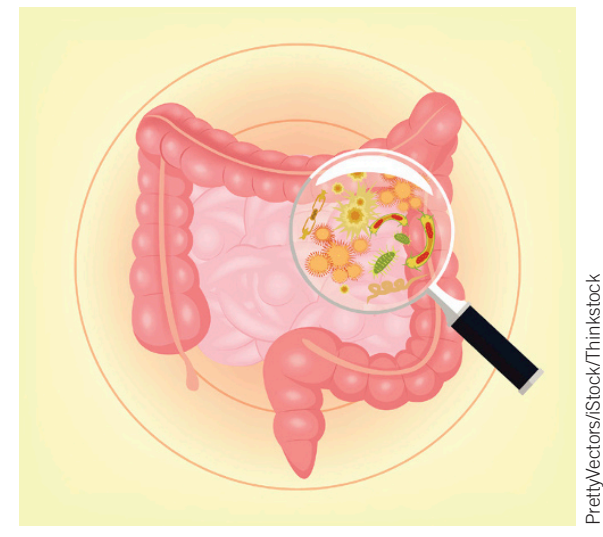

Each diet profoundly changed the composition of the microbiota. Bilophila spp. bacteria were more prevalent in mice that were fed lard, which is consistent with prior reports that describe the presence of similar bacteria in humans fed a diet rich in saturated fats.

To experimentally test whether changes in a microbiota directly contribute to disease, researchers can transfer the microbiota from a donor into a recipient mouse that lacks a microbiota. In this study, recipients were treated with antibiotics to deplete the microbes in their gut; they were then colonized with the microbiota from mice fed either lard or fish oil. All recipient mice were subsequently fed the lard diet. Over 3 weeks, mice that received microbiota from mice that were fed fish oil gained less weight and had less inflammation in fat tissue than other recipient mice. This suggests that their donated microbiota conferred protection against a diet rich in saturated fats.

In both donors and colonized recipients of this beneficial microbiota, the bacteria Akkermansia muciniphila was notably abundant. A. muciniphila has previously been shown to contribute to protection against diet-induced obesity, and these findings support the investigation of new probiotics or prebiotics to treat metabolic disease. However, in a press release, Fredrik Bäckhed clarifies that "further investigations will be needed to determine if these bacteria can be used as probiotic strain and, in that case, how it should be combined with diet to optimize health outcomes."

\section{Kevin Da Silva}

\section{IMPORT/EXPORT TMBALANCE_IN ALS}

Amyotrophic lateral sclerosis (ALS), also called Lou Gehrig's disease, is a neurodegenerative disease that leads to paralysis and ultimately death in almost all cases. About $10 \%$ of cases are familial, and mutations in the gene C90RF72 are to blame for about $40 \%$ of inherited cases. The function of C9ORF72 was unknown when it was discovered, but recently, three papers have identified a molecular mechanism that explains its role in causing ALS.

The three studies (Nature 525, 56-61 and 129-133; and Nat. Neurosci. 18, 1226-1229; 2015) used unbiased screens in Drosophila melanogaster and yeast to identify genes that interacted with C9ORF72 in causing ALS-related pathology. These screens consistently identified proteins that function to regulate the import of other proteins into the cell nucleus.

The C9ORF72 gene contains a section of DNA in which a six-nucleotide pattern repeats. "Healthy people have two to five repeats of this six-nucleotide pattern," said Aaron Gitler (Stanford University, Palo Alto, CA), senior author on the paper in Nature Neuroscience, in a press release. "But in some people, this region is expanded into hundreds or thousands of copies." To figure out how this repeat expansion leads to ALS pathology, the authors of the three studies engineered Drosophila or yeast expressing the expanded gene, which caused toxicity in yeast and fly cells, and locomotor deficits in flies. The researchers then screened hundreds of genes, seeking those that affect this toxicity.

In each study, the screens independently identified proteins involved in the import of nuclear proteins. Genes that increased nuclear import or suppressed nuclear export of proteins ameliorated neurodegeneration, whereas genes that interfered with nuclear import exacerbated it. These results were confirmed in neurons derived from patients that carry the C90RF72 mutation. Postmortem brain tissue from ALS patients with the mutation also showed deficits in nuclear import of proteins. "Combining a simple fruit fly model with experiments in cells donated by ALS and [frontotemporal dementia] patients was essential for discovering the disease mechanism underlying mutations in C9ORF72," said Paul Taylor (St. Jude Children's Research Hospital, Memphis, TN), senior author of one of the papers in Nature, in a press release. The other paper in Nature showed that a drug that interferes with the interaction between nuclear import proteins and RNA from repeat-expanded C9ORF72 positively affected pathology in Drosophila. "This is certainly a good therapeutic target," said Jeff Rothstein (Johns Hopkins University Medical School, Baltimore, MD), the paper's senior author.

Brigitta B. Gundersen 